Applied Mathematical Sciences, Vol. 9, 2015, no. 10, 493 - 505

HIKARI Ltd, www.m-hikari.com

http://dx.doi.org/10.12988/ams.2015.410786

\title{
Solutions of Generalized Linear Matrix Differential Equations which Satisfy Boundary Conditions at Two Points
}

\author{
Charalambos P. Kontzalis and Panayiotis Vlamos \\ Department of Informatics \\ Ionian University, Corfu, Greece
}

Copyright (c) 2014 Charalambos P. Kontzalis and Panayiotis Vlamos. This is an open access article distributed under the Creative Commons Attribution License, which permits unrestricted use, distribution, and reproduction in any medium, provided the original work is properly cited.

\begin{abstract}
In this article, we study a boundary value problem of a class of generalized linear matrix differential equations whose coefficients are square constant matrices. By using matrix pencil theory we obtain formulas for the solutions and we give necessary and sufficient conditions for existence and uniqueness of solutions. Moreover we provide some numerical examples. These kinds of systems are inherent in many physical and engineering phenomena.
\end{abstract}

Keywords: linear differential equations, boundary value problem, singular, system

\section{Introduction}

Many authors have studied generalized continuous \& discrete time systems, see $[3-6,10-12,14-16,19,23,24,29-34,37-39,44-46]$, and their applications, see $[2,3,20-22,25,26,48-50,53,54]$. Many of these results have already been extended to systems of differential \& difference equations with fractional operators, see $[13,17,18,20,24,25,33-36,40-42]$. In this article, our purpose is to study the solutions of a generalized boundary value problem of Linear Matrix Differential Equations (LMDEs) into the mainstream of matrix pencil 
theory. A boundary-value problem consists of finding solutions which satisfies an ordinary matrix differential equation and appropriate boundary conditions at two or more points. Thus we consider the boundary value problem

$$
F Y^{\prime}(t)=G Y(t), \quad A Y\left(t_{0}\right)=D, \quad B Y\left(t_{N}\right)=E .
$$

where $F, G, A, B \in \mathcal{M}(m \times m ; \mathcal{F})$ and $Y(t), D, E \in \mathcal{M}(m \times 1 ; \mathcal{F})$, (i.e. the algebra of square matrices with elements in the field $\mathcal{F}$ ). For the sake of simplicity we set $\mathcal{M}_{m}=\mathcal{M}(m \times m ; \mathcal{F})$ and $\mathcal{M}_{n m}=\mathcal{M}(n \times m ; \mathcal{F})$. Systems of type (1) are more general, including the special case when $F=I_{n}$, where $I_{n}$ is the identity matrix of $\mathcal{M}_{n}$.

\section{Mathematical Background and Notation}

This brief subsection introduces some preliminary concepts and definitions from matrix pencil theory, which are being used throughout the paper. Linear systems of type (1) are closely related to matrix pencil theory, since the algebraic geometric, and dynamic properties stem from the structure by the associated pencil $s F-G$. Given $F, G \in M_{n m}$ and an indeterminate $s \in \mathcal{F}$, the matrix pencil $s F-G$ is called regular when $m=n$ and $\operatorname{det}(s F-G) \neq 0$, see $[10,28,38,43,47]$. In any other case, the pencil will be called singular. In addition, the pencil $s F-G$ is said to be strictly equivalent to the pencil $s \tilde{F}-\tilde{G}$ if and only if there exist non-singular $P \in \mathcal{M}_{m}$ and $Q \in \mathcal{M}_{m}$ such as

$$
P(s F-G) Q=s \tilde{F}-\tilde{G} .
$$

In this article, we consider the case that pencil is regular. The class of $s F-G$ is characterized by a uniquely defined element, known as a complex Weierstrass canonical form, $s F_{w}-G_{w}$, see $[10,28,38,43,47]$, specified by the complete set of invariants of the pencil $s F-G$. This is the set of elementary divisors (e.d.) obtained by factorizing the invariant polynomials $f_{i}(s, \hat{s})$ into powers of homogeneous polynomials irreducible over field $F$. In the case where $s F-G$ is a regular, we have e.d. of the following type:

- e.d. of the type $s^{p}$ are called zero finite elementary divisors (z. f.e.d.)

- e.d. of the type $(s-a)^{\pi}, a \neq 0$ are called nonzero finite elementary divisors (nz. f.e.d.)

- e.d. of the type $\hat{s}^{q}$ are called infinite elementary divisors (i.e.d.).

Let $B_{1}, B_{2}, \ldots, B_{n}$ be elements of $\mathcal{M}_{n}$. The direct sum of them denoted by $B_{1} \oplus B_{2} \oplus \ldots \oplus B_{n}$ is the block $\operatorname{diag}\left\{B_{1}, B_{2}, \ldots, B_{n}\right\}$. Then, the complex 
Weierstrass form $s F_{w}-G_{w}$ of the regular pencil $s F-G$ is defined by $s F_{w}-$ $G_{w}:=s I_{p}-J_{p} \oplus s H_{q}-I_{q}$, where the first normal Jordan type element is uniquely defined by the set of f.e.d.

$$
\left(s-a_{1}\right)^{p_{1}}, \ldots,\left(s-a_{\nu}\right)^{p_{\nu}}, \quad \sum_{j=1}^{\nu} p_{j}=p
$$

of $s F-G$ and has the form

$$
s I_{p}-J_{p}:=s I_{p_{1}}-J_{p_{1}}\left(a_{1}\right) \oplus \ldots \oplus s I_{p_{\nu}}-J_{p_{\nu}}\left(a_{\nu}\right) .
$$

And also the $q$ blocks of the second uniquely defined block $s H_{q}-I_{q}$ correspond to the i.e.d.

$$
\hat{s}^{q_{1}}, \ldots, \hat{s}^{q_{\sigma}}, \quad \sum_{j=1}^{\sigma} q_{j}=q
$$

of $s F-G$ and has the form

$$
s H_{q}-I_{q}:=s H_{q_{1}}-I_{q_{1}} \oplus \ldots \oplus s H_{q_{\sigma}}-I_{q_{\sigma}} .
$$

Thus, $H_{q}$ is a nilpotent element of $\mathcal{M}_{n}$ with index $\tilde{q}=\max \left\{q_{j}: j=1,2, \ldots, \sigma\right\}$, then

$$
H_{q}^{\tilde{q}}=0_{q, q} \cdot
$$

We denote with $O_{q, q}$ the zero matrix. $I_{p_{j}}, J_{p_{j}}\left(a_{j}\right), H_{q_{j}}$ are defined as

$$
J_{p_{j}}\left(a_{j}\right)=\left[\begin{array}{ccccc}
a_{j} & 1 & \ldots & 0 & 0 \\
0 & a_{j} & \ldots & 0 & 0 \\
\vdots & \vdots & \ddots & \vdots & \vdots \\
0 & 0 & \ldots & a_{j} & 1 \\
0 & 0 & \ldots & 0 & a_{j}
\end{array}\right] \in \mathcal{M}_{p_{j}}, H_{q_{j}}=\left[\begin{array}{ccccc}
0 & 1 & \ldots & 0 & 0 \\
0 & 0 & \ldots & 0 & 0 \\
\vdots & \vdots & \ddots & \vdots & \vdots \\
0 & 0 & \ldots & 0 & 1 \\
0 & 0 & \ldots & 0 & 0
\end{array}\right] \in \mathcal{M}_{q_{j}}
$$

\section{Main Results-Solution space form of a con- sistent boundary value problem}

In this section, the main results for a consistent boundary value problem of type (1) are analytically presented. Moreover, it should be stressed out that these results offer the necessary mathematical framework for interesting applications.

Definition 2.1. The boundary value problem (1) is said to be consistent if it possesses at least one solution. 
Consider the problem (1). From the regularity of $s F-G$, there exist nonsingular $\mathcal{M}(m \times m, F)$ matrices $P$ and $Q$ such that (see also Section 1 ),

$$
P F Q=F_{w}=I_{p} \oplus H_{q}, \quad P G Q=G_{w}=J_{p} \oplus I_{q}
$$

Where $I_{p_{j}}, J_{p_{j}}\left(a_{j}\right), H_{q_{j}}$ are defined in Section 1 .

Lemma 2.1. System (1) is divided into two subsystems:

$$
Z_{p}^{\prime}(t)=J_{p} Z_{p}(t)
$$

and the subsystem

$$
H_{q} Z_{q}^{\prime}(t)=Z_{q}(t)
$$

Proof. Consider the transformation

$$
Y(t)=Q Z(t)
$$

Substituting the previous expression into (1) we obtain

$$
F Q Z^{\prime}(t)=G Q Z(t)
$$

where by multiplying by $P$ and using (2) we arrive at

$$
F_{w} Z^{\prime}(t)=G_{w} Z(t)
$$

Moreover, we can write $Z(t)$ as $Z(t)=\left[\begin{array}{c}Z_{p}(t) \\ Z_{q}(t)\end{array}\right]$, where $Z_{p}(t) \in \mathcal{M}_{p 1}$ and $Z_{q}(t) \in \mathcal{M}_{q 1}$. Taking into account the above expressions, we arrive easily at (3) and (4).

From $[1,7,27,48-52]$ it is known that the subsystem (3) has the general solution

$$
Z_{p}(t)=e^{J_{p}\left(t-t_{0}\right)} C,
$$

where $\sum_{j=1}^{v} p_{j}=p$ and $C \in \mathcal{M}_{m 1}$ constant.

Proposition 2.1. The subsystem (4) has the unique solution

$$
Z_{q}(t)=0_{q, q} .
$$

Proof. Let $q_{*}$ be the index of the nilpotent matrix $H_{q}$, i.e. $\left(H_{q}^{q_{*}}=0_{q, q}\right)$, we obtain the following equations

$$
\begin{gathered}
H_{q} Z_{q}^{\prime}(t)=Z_{q}(t), \\
H_{q}^{2} Z_{q}^{\prime}(t)=H_{q} Z_{q}(t), \\
H_{q}^{3} Z_{q}^{\prime}(t)=H_{q}^{2} Z_{q}(t), \\
\vdots \\
H_{q}^{q_{*}} Z_{q}^{\prime}(t)=H_{q}^{q_{*}-1} Z_{q}(t)
\end{gathered}
$$


and

$$
\begin{gathered}
H_{q} Z_{q}^{\prime}(t)=Z_{q}(t), \\
H_{q}^{2} Z_{q}^{\prime \prime}(t)=H_{q} Z_{q}^{\prime}(t), \\
H_{q}^{3} Z_{q}^{\prime \prime \prime}(t)=H_{q}^{2} Z_{q}^{\prime \prime}(t), \\
\vdots \\
H_{q}^{q_{*}} Z_{q}^{\left(q_{*}\right)}(t)=H_{q}^{\left(q_{*}-1\right)} Z_{q}(t) .
\end{gathered}
$$

The sum of the above relations gives, (note $H_{q}^{q_{*}}=0_{q q}$ ) is the solution (6).

\section{The boundary value problem}

A necessary and sufficient condition for the boundary value problem to be consistent is given by the following result

Theorem 2.1. The boundary value problem (1) is consistent, if and only if

$$
D, E \in \operatorname{colspan}\left[A Q_{p}\right]=\operatorname{col} \operatorname{span}\left[B Q_{p} e^{J_{p}\left(t_{N}-t_{0}\right)}\right]
$$

Where $Q_{p}$ has column vectors all the linear independent eigenvectors of the finite generalized eigenvalues of $s F-G$.

Proof. Let $Q=\left[Q_{p} Q_{q}\right]$, where $Q_{p} \in \mathcal{M}_{m p}$ and $Q_{q} \in \mathcal{M}_{m q}$. Combining (5), (5), we obtain

$$
Y(t)=Q Z(t)=\left[Q_{p} Q_{q}\right]\left[\begin{array}{c}
Q_{p} e^{J_{p}\left(t-t_{0}\right)} C \\
0_{q 1}
\end{array}\right]
$$

and

$$
Y(t)=Q_{p} e^{J_{p}\left(t-t_{0}\right)} C .
$$

This solution exists if and only if

$$
\begin{aligned}
& D=A Y\left(t_{0}\right) \\
& E=B Y\left(t_{N}\right)
\end{aligned}
$$

and

$$
\begin{gathered}
D=A Q_{p} C \\
E=B Q_{p} e^{J_{p}\left(t_{N}-t_{0}\right)} C,
\end{gathered}
$$

or,

$$
D, E \in \operatorname{col} \operatorname{span}\left[A Q_{p}\right]=\operatorname{col} \operatorname{span}\left[B Q_{p} e^{J_{p}\left(t_{N}-t_{0}\right)}\right] .
$$

The columns of $Q_{p}$ are the $p$ eigenvectors of the finite elementary divisors (eigenvalues) of the pencil $s F-G$. (see [9, 25, 35, 40, 44, 48] for algorithms 
for the computation of $Q_{p}$ )

It is obvious that, if there is a solution of the boundary value problem, it needs not to be unique. The necessary and sufficient conditions, for uniqueness, when the problem is consistent, are given by the following theorem.

Theorem 2.2 Assume the boundary value problem (1). Then when it is consistent, it has a unique solutions if and only

$$
\operatorname{rank}\left[A Q_{p}\right]=\operatorname{rank}\left[B Q_{p} e^{J_{p}\left(t_{N}-t_{0}\right)}\right]=p
$$

and the linear system

$$
\begin{gathered}
A Q_{p} C=D \\
B Q_{p} e^{J_{p}\left(t_{N}-t_{0}\right)} C=E
\end{gathered}
$$

gives a unique solution for constant column $C$. Then the unique solution is given by

$$
Y(t)=Q_{p} e^{J_{p}\left(t-t_{0}\right)} C
$$

Proof Let the boundary value problem (1) be consistent, then from Theorem 2.1 the solution is

$$
Y(t)=Q_{p} e^{J_{p}\left(t-t_{0}\right)} C
$$

with

$$
\begin{aligned}
& D=A Y\left(t_{0}\right) \\
& E=B Y\left(t_{N}\right)
\end{aligned}
$$

and

$$
\begin{gathered}
D=A Q_{p} C \\
E=B Q_{p} e^{J_{p}\left(t_{N}-t_{0}\right)} C .
\end{gathered}
$$

It is clear that for given $A, B, D, E$ the problem (1) has a unique solution if and only if the system (9) has a unique solution. Since $A Q_{p}, B Q_{p} e^{J_{p}\left(t-t_{0}\right)} \in$ $\mathcal{M}_{m p}$, the solution is unique for the system (9) if and only if the matrices $A Q_{p}, B Q_{p} e^{J_{p}\left(t-t_{0}\right)}$ are left invertible $\left(\operatorname{rank}\left[A Q_{p}\right]=\operatorname{rank}\left[B Q_{p} e^{J_{p}\left(t-t_{0}\right)}\right]=p\right)$ and both equations give the same unique solution for the constant column $C$. 


\section{Numerical Example}

Consider the boundary value problem (1). Where

$$
F=\left[\begin{array}{llllll}
1 & 0 & 0 & 0 & 0 & 0 \\
0 & 1 & 0 & 0 & 0 & 0 \\
0 & 0 & 1 & 0 & 0 & 0 \\
0 & 0 & 0 & 1 & 0 & 0 \\
0 & 0 & 0 & 0 & 1 & 1 \\
0 & 0 & 0 & 0 & 0 & 0
\end{array}\right], G=\left[\begin{array}{cccccc}
0 & 0 & 1 & 0 & 0 & 0 \\
0 & 0 & 0 & 1 & 0 & 0 \\
0 & 0 & 0 & 0 & 1 & 0 \\
0 & 0 & 0 & 0 & 0 & 1 \\
-4 & 2 & 2 & -3 & -2 & -1 \\
1 & 1 & -1 & -1 & 0 & 0
\end{array}\right]
$$

and $A$ the identity matrix and and $B$ a matrix that satisfies the equation $B Q_{p} e^{J_{p}\left(t_{N}-t_{0}\right.}=Q_{p}$. The invariants of $s F-G$ are $s-1, s-2, s-3$ (finite elementary divisors) and $\bar{s}^{3}$ (infinte elementary divisor of degree 3 ). Then

$$
J_{3}=\left[\begin{array}{lll}
1 & 0 & 0 \\
0 & 2 & 0 \\
0 & 0 & 3
\end{array}\right]
$$

and the columns of $Q_{p}$ are the eigenvectors of the generalized eigenvalues 1, 2, 3 respectively. Then

$$
A Q_{p}=B Q_{p} e^{J_{p}\left(t_{N}-t_{0}\right)}=\left[\begin{array}{cccccc}
3 & -5 & 3 & -5 & 3 & -5 \\
1 & -1 & 2 & -2 & 4 & -4 \\
1 & -1 & 3 & -3 & 9 & -9
\end{array}\right]
$$

\section{Example 1}

Let

$$
D=E=\left[\begin{array}{llllll}
1 & -3 & -2 & 0 & -10 & 8
\end{array}\right]^{T}
$$

Then

$$
D, E \in \operatorname{colspan}\left[A Q_{p}\right]=\mathrm{col} \operatorname{span}\left[B Q_{p} e^{J_{p}\left(t_{N}-t_{0}\right)}\right]
$$

and by calculating $C$ from (9) we get

$$
C=\left[\begin{array}{lll}
1 & -1 & -1
\end{array}\right]^{T}
$$

and the unique solution of the system by substituting in (10) is

$$
Y(t)=\left[\begin{array}{c}
3 e^{t}-e^{2 t}-e^{3 t} \\
-5 e^{t}+e^{2 t}+e^{3 t} \\
3 e^{t}-2 e^{2 t}-3 e^{3 t} \\
-5 e^{t}+2 e^{2 t}+3 e^{3 t} \\
3 e^{t}-4 e^{2 t}-9 e^{2 t} \\
-5 e^{t}+4 e^{2 t}+9 e^{3 t}
\end{array}\right]
$$




\section{Example 2}

Let

$$
D=\left[\begin{array}{llllll}
0 & 0 & 0 & 0 & 1 & 1
\end{array}\right]^{T}
$$

Then

$$
D \notin \operatorname{colspan}\left[A Q_{p}\right]=\operatorname{colspan}\left[B Q_{p} e^{J_{p}\left(t_{N}-t_{0}\right)}\right]
$$

and thus the boundary value problem is not consistent.

\section{Acknowledgement}

The authors would like to express their sincere gratitude to Professor G.I. Kalogeropoulos for his helpful and fruitful discussions that led to necessary changes and modifications in the proof of the theorems. Moreover, we are very grateful to the anonymous referees for their valuable suggestions that clearly improved this article.

\section{Conclusions}

The aim of this article was to give necessary and sufficient conditions for existence and uniqueness of solutions for generalized linear discrete time boundary value problems of a class of linear rectangular matrix differential equations whose coefficients are square constant matrices. By taking into consideration that the relevant pencil is regular, we get effected by the Weierstrass canonical form in order to decompose the differential system into two sub-systems. Afterward, we provide analytic formulas when we have a consistent problem. Moreover, as a further extension of the present paper, we can discuss the case where the pencil is singular. Thus, the Kronecker canonical form is required. For all these, there is some research in progress.

\section{References}

[1] T. M. Apostol; Explicit formulas for solutions of the second order matrix differential equation $Y^{\prime \prime}=A Y$, Amer. Math. Monthly 82 (1975), pp. 159162. http://dx.doi.org/10.2307/2319663

[2] R. Ben Taher and M. Rachidi; Linear matrix differential equations of higher-order and applications, E. J. of Differential Eq., Vol. 2008 (2008), No. 95, pp. 1-12. 
[3] S. L. Campbell, C.D. Meyer and N.J. Rose ; Applications of the Drazin inverse to linear systems of differential equations with singular constant coefficients, SIAM J. Appl. Math. 31(3) 411-425, (1976). http://dx.doi.org/10.1137/0131035

[4] S. L. Campbell; Comments on 2-D descriptor systems, Automatica (Journal of IFAC), v.27 n.1, p.189-192. (1991). http://dx.doi.org/10.1016/00051098(91)90020-3

[5] S. L. Campbell; Singular systems of differential equations, Pitman, San Francisco, Vol. 1, 1980; Vol. 2, 1982. http://dx.doi.org/10.1002/nme.1620150916

[6] S. L. Campbell; Singular systems of differential equations, Pitman, San Francisco, Vol. 1, 1980; Vol. 2, 1982. http://dx.doi.org/10.1002/nme.1620150916

[7] H.-W. Cheng and S. S.-T. Yau; More explicit formulas for the matrix exponential, Linear Algebra Appl. 262 (1997), pp. 131-163. http://dx.doi.org/10.1016/s0024-3795(97)80028-6

[8] B.N. Datta; Numerical Linear Algebra and Applications, Cole Publishing Company, 1995.

[9] L. Dai, Singular Control Systems, Lecture Notes in Control and information Sciences Edited by M.Thoma and A.Wyner (1988). http://dx.doi.org/10.1007/bfb0002475

[10] L. Dai, Impulsive modes and causality in singular systems, International Journal of Control, Vol 50, number 4 (1989). http://dx.doi.org/10.1080/00207178908953432

[11] I.K. Dassios, On non-homogeneous linear generalized linear discrete time systems, Circuits systems and signal processing, Volume 31, Number 5, 1699-1712 (2012). http://dx.doi.org/10.1007/s00034-012-9400-7

[12] I.K. Dassios, G. Kalogeropoulos, On a non-homogeneous singular linear discrete time system with a singular matrix pencil, Circuits systems and signal processing (2013). http://dx.doi.org/10.1007/s00034-012-9541-8

[13] I.K. Dassios, Optimal solutions for non-consistent singular linear systems of fractional nabla difference equations, Circuits systems and signal processing, (2014). DOI 10.1007/s00034-014-9930-2

[14] I. Dassios, On solutions and algebraic duality of generalized linear discrete time systems, Discrete Mathematics and Applications, Volume 22, No. 56, 665-682 (2012). http://dx.doi.org/10.1515/dma-2012-045 
[15] I. Dassios, On stability and state feedback stabilization of singular linear matrix difference equations, Advances in difference equations, 2012:75 (2012). http://dx.doi.org/10.1186/1687-1847-2012-75

[16] I. Dassios, On robust stability of autonomous singular linear matrix difference equations, Applied Mathematics and Computation, Volume 218, Issue 12, 6912-6920 (2012). http://dx.doi.org/10.1016/j.amc.2011.12.067

[17] I.K. Dassios, D. Baleanu, On a singular system of fractional nabla difference equations with boundary conditions, Boundary Value Problems, 2013:148 (2013). http://dx.doi.org/10.1186/1687-2770-2013-148

[18] I. Dassios, D. Baleanu, G. Kalogeropoulos, On non-homogeneous singular systems of fractional nabla difference equations, Applied Mathematics and Computation, Volume 227, 112-131 (2014). http://dx.doi.org/10.1016/j.amc.2013.10.090

[19] I. Dassios, G. Kalogeropoulos, On the relation between consistent and non consistent initial conditions of singular discrete time systems, Dynamics of continuous, discrete and impulsive systems Series A: Mathematical Analysis, Volume 20, Number 4a, pp. 447-458 (2013).

[20] I. Podlubny, Fractional Differential Equations, Mathematics in Science and Engineering,p. xxiv+340. Academic Press, San Diego, Calif, USA (1999).

[21] A. P. Schinnar, The Leontief dynamic generalized inverse. The Quarterly Journal of Economics 92.4 pp. 641-652 (1978). http://dx.doi.org/10.2307/1883180

[22] I. Dassios, G. Kalogeropoulos, On the stability of equilibrium for a reformulated foreign trade model of three countries. Journal of Industrial Engineering International, Springer, (2014) 10:71 DOI 10.1007/s40092014-0071-9.

[23] I. Dassios, On a boundary value problem of a class of generalized linear discrete time systems, Advances in Difference Equations, Springer, 2011:51 (2011). http://dx.doi.org/10.1186/1687-1847-2011-51

[24] F. M. Atici, P. W. Eloe, Initial value problems in discrete fractional calculus, Proceedings of the American Mathematical Society, vol. 137, no. 3, pp. 981-989 (2009). http://dx.doi.org/10.1090/s0002-9939-08-09626-3

[25] D. Baleanu, K. Diethelm, E. Scalas, Fractional Calculus: Models and Numerical Methods, World Scientific (2012). http://dx.doi.org/10.1142/8180 
[26] I. Dassios, A. Zimbidis, The classical Samuelson's model in a multicountry context under a delayed framework with interaction, Dynamics of continuous, discrete and impulsive systems Series B: Applications \& Algorithms, Volume 21, Number 4-5b pp. 261-274 (2014).

[27] G. Doetsch, Introduction to the theory and application of the Laplace transformation, Springer-Verlag, 1974. http://dx.doi.org/10.1007/978-3642-65690-3

[28] R. F. Gantmacher; The theory of matrices I, II, Chelsea, New York, (1959). http://dx.doi.org/10.1126/science.131.3408.1216-a

[29] E. Grispos, S. Giotopoulos, G. Kalogeropoulos; On generalised linear discrete-time regular delay systems., J. Inst. Math. Comput. Sci., Math. Ser. 13, No.2, 179-187, (2000).

[30] E. Grispos, G. Kalogeropoulos, M. Mitrouli, On generalised linear discrete-time singular delay systems., Lipitakis, Elias A. (ed.), HERCMA 2001. Proceedings of the 5th Hellenic-European conference on computer mathematics and its applications, Athens, Greece, September 20-22, 2001. 2 volumes. Athens: LEA. 484-486 (2002).

[31] E. Grispos, G. Kalogeropoulos, I. Stratis, On generalised linear discretetime singular delay systems., J. Math. Anal. Appl. 245, No.2, 430-446 (2000). http://dx.doi.org/10.1006/jmaa.2000.6761

[32] E. Grispos, Singular generalised autonomous linear differential systems., Bull. Greek Math. Soc. 34, 25-43 (1992).

[33] Kaczorek, T.; General response formula for two-dimensial linear systems with variable coefficients. IEEE Trans. Aurom. Control Ac-31, 278-283, (1986). http://dx.doi.org/10.1109/tac.1986.1104238

[34] Kaczorek, T.; Equivalence of singular 2-D linear models. Bull. Polish Academy Sci., Electr. Electrotechnics, 37, (1989).

[35] T. Kaczorek, Selected problems of fractional systems theory. Vol. 411. Springer (2011).

[36] T. Kaczorek, Application of the Drazin inverse to the analysis of descriptor fractional discrete-time linear systems with regular pencils. Int. J. Appl. Math. Comput. Sci 23.1, 2013: 29-33 (2014). http://dx.doi.org/10.2478/amcs-2013-0003

[37] C. Kontzalis, G. Kalogeropoulos. Controllability and reachability of singular linear discrete time systems. arXiv preprint arXiv:1406.1489 (2014). 
[38] G. I. Kalogeropoulos; Matrix pencils and linear systems, Ph.D Thesis, City University, London, (1985).

[39] G. Kalogeropoulos, I.G. Stratis; On generalized linear regular delay systems., J. Math. Anal. Appl. 237, No.2, 505-514, (1999). http://dx.doi.org/10.1006/jmaa.1999.6458

[40] J. Klamka, J. Wyrwa, Controllability of second-order infinitedimensional systems. Syst. Control Lett. 57, No. 5, 386-391 (2008). http://dx.doi.org/10.1016/j.sysconle.2007.10.002

[41] J. Klamka, Controllability and minimum energy control problem of fractional discrete-time systems, Chapter in monograph New Trends in Nanotechnology and Fractional Calculus. Springer-Verlag. New York. pp. 503509 (2010). http://dx.doi.org/10.1007/978-90-481-3293-5_45

[42] J. Klamka, Controllability of dynamical systems, Matematyka Stosowana, 50, no.9, pp.57-75, (2008).

[43] I. E. Leonard; The matrix exponential, SIAM Review Vol. 38, No. 3 (1996), pp. 507-512. http://dx.doi.org/10.1137/s0036144595286488

[44] F. L. Lewis; A survey of linear singular systems, Circuits Syst. Signal Process. 5, 3-36, (1986). http://dx.doi.org/10.1007/bf01600184

[45] F.L. Lewis; Recent work in singular systems, Proc. Int. Symp. Singular systems, pp. 20-24, Atlanta, GA, (1987).

[46] F. L. Lewis; A review of $2 D$ implicit systems, Automatica (Journal of IFAC), v.28 n.2, p.345-354, (1992). http://dx.doi.org/10.1016/00051098(92)90120-5

[47] M. Mitrouli, G. Kalogeropoulos; A compound matrix algorithm for the computation of the Smith form of a polynomial matrix., Numer. Algorithms 7, no. 2-4, 145-159, (1994). http://dx.doi.org/10.1007/bf02140679

[48] Ogata, K: Discrete Time Control Systems. Prentice Hall, (1987).

[49] W.J. Rugh; Linear system theory, Prentice Hall International (Uk), London (1996).

[50] J.T. Sandefur; Discrete Dynamical Systems, Academic Press, (1990).

[51] G.W. Steward and J.G. Sun; Matrix Perturbation Theory, Oxford University Press, (1990). 
[52] L. Verde-Star; Operator identities and the solution of linear matrix difference and differential equations, Studies in Applied Mathematics 91 (1994), pp. $153-177$.

[53] D.N. Vizireanu; A fast, simple and accurate timevarying frequency estimation method for single-phase electric power systems, Measurement 45, (5), 1331-3, (2012). http://dx.doi.org/10.1016/j.measurement.2012.01.038

[54] D.N. Vizireanu, S.V. Halunga, Simple, fast and accurate eight points amplitude estimation method of sinusoidal signals for DSP based instrumentation, Journal of Instrumentation, 7(4), (2012). http://dx.doi.org/10.1088/1748-0221/7/04/p04001

Received: October 15, 2014; Published: January 7, 2015 